\title{
Editorial \\ Single-Cell RNA Sequencing Uncovers Heterogeneous Circulating Tumor Cell Subsets in Breast Cancer
}

\author{
Maria A. Papadaki ${ }^{1}$ (D) and Sofia Agelaki ${ }^{1,2, *}$ \\ 1 Laboratory of Translational Oncology, School of Medicine, University of Crete, 70013 Heraklion, Greece; \\ papadaki_maria1@yahoo.gr \\ 2 Department of Medical Oncology, University General Hospital of Heraklion, 71110 Heraklion, Greece \\ * Correspondence: agelakisofia@gmail.com; Tel.: +30-2810-394712
}

Citation: Papadaki, M.A.; Agelaki, S. Single-Cell RNA Sequencing

Uncovers Heterogeneous Circulating Tumor Cell Subsets in Breast Cancer. Cancers 2022, 14, 1314. https:// doi.org/10.3390/cancers14051314

Received: 1 March 2022

Accepted: 2 March 2022

Published: 4 March 2022

Publisher's Note: MDPI stays neutral with regard to jurisdictional claims in published maps and institutional affiliations.

Copyright: (c) 2022 by the authors. Licensee MDPI, Basel, Switzerland. This article is an open access article distributed under the terms and conditions of the Creative Commons Attribution (CC BY) license (https:// creativecommons.org/licenses/by/ $4.0 /)$.

\section{Introduction}

Metastasis remains the main cause of death for breast cancer (BC) patients, and conceivably, a huge effort has been directed toward the understanding of the metastatic process [1]. Circulating tumor cells (CTCs), identified in the peripheral blood (PB) of patients with $\mathrm{BC}$ and other solid tumors, have been recognized as important precursors of metastasis [2]. Accordingly, their detection represents a valuable prognostic biomarker predicting poor survival measures in $\mathrm{BC}$, and at the same time, it holds a promising role in monitoring disease progression [3]. Importantly, CTC analysis offers the unique opportunity to explore the biological characteristics of tumor cells that participate in the formation of metastases, thus expanding our knowledge on the mechanisms underlying disease progression [4]. Finally, CTC analysis can inform on the molecular characteristics of the tumor in real time, which could serve as an important tool for guiding personalized therapy in BC [5].

Recently, cutting-edge methodologies, such as RNA sequencing (RNA-seq) and singlecell RNA-seq (scRNA-seq), are increasingly being used to uncover the specific biological features of CTCs that drive metastatic progression and resistance to therapy. Specifically in BC, Boral D. et al. utilized whole-genome mRNA microarray and pathway analyses to show that CTCs from patients with documented brain metastases demonstrated enhanced activation of Notch signaling and increased pro-inflammatory chemokines, immunomodulatory networks, and mitogenic growth factors [6]. More recently, transcriptomic analysis of CTCs from triple-negative breast cancer (TNBC) patients revealed a liver metastasisassociated TNBC gene signature, which uncovered valuable prognostic and drug candidate biomarkers for TNBC [7]. scRNA-seq of CTCs has also been employed in other malignancies, such as multiple myeloma, hepatocellular, pancreatic, and prostate cancer, offering critical insights into the biology of metastasis and the mechanisms of resistance [8-11].

However, in most of these studies, RNA-seq analysis was restricted to CTCs selected according to their "classical" definition, which in general requires positivity for epithelial markers, such as cytokeratins (CKs) and epithelial-cell adhesion molecule (EpCAM), along with negativity for the common lymphocytic marker CD45 [12]. It should be noted here that this definition has been consistently used for the detection and enumeration of CTCs, providing significant prognostic information for patients with different types of cancer, including BC [2]. However, it is also widely accepted that CTCs constitute a highly heterogeneous cell population, which can frequently lose epithelial marker expression in the context of epithelial-to-mesenchymal transition (EMT) [13]. Especially in BC, a growing body of evidence supports the notion that CTCs bearing semi-EMT-like, or even fully mesenchymal-like phenotypes, also demonstrate cancer stem cell (CSC) properties and enhanced survival, immune evasion, and metastatic potential, as well as resistance to conventional therapies $[6,14-16]$. Thus, it is critical to appreciate that the analysis of 
the entire CTC compartment is critical to delineate CTC heterogeneity in order to uncover specific tumor characteristics that drive metastatic progression.

\section{The Study by Pauken M.C. et al., Published in Cancers}

Pauken M.C. and co-authors [17], in an effort to describe the whole spectrum of CTC subsets that contribute to CTC heterogeneity and metastatic potency, utilized the transcriptomic analysis of white blood cells from 21 metastatic BC patients. Their experimental strategy included the isolation of cells based on lineage expression through flow cytometry, followed by analysis using the cutting-edge technologies of RNA-seq and scRNA-seq. Through the interrogation of both the Lineage- and Lineage+ (Lin-/Lin+) cells, the study revealed a "CTC-candidate" cell cluster harboring distinct gene expression patterns that subjects the classical definition of CTCs to revision and highlights the importance of exploring the properties of the entire population of CTCs in order to gain further insights into the putative mechanisms of metastasis.

More specifically, the initial RNA-seq analysis revealed a high number of differentially expressed genes (DEGs) between the Lin+ and Lin - cell populations. The Lin+ cell population was enriched with genes suggestive of an immune cell phenotype, such as PTPRC (CD45), CD3, CD4, CD8, CD27, and HEY1). Among the most significant LinDEGs identified were CAVIN2, ITGB3, LY6G6F, TUBB1, LTBP1, and TRIM58, as well as genes suggestive of an epithelial phenotype (e.g., EPCAM, TACSTD2, MUC2, KRT7, 8, 18, and 19), and genes specific to mammary tissue (e.g., LTF and CTTN). Further analysis of these genes revealed the enrichment of several pathways, including EMT, apical junctions, focal adhesion, estrogen response, and angiogenesis. These findings implied that the epithelial/mammary cells were enriched in the Lin-population.

To further delineate individual differences in gene expression between Lin - and Lin+ subsets, scRNA-seq analysis was indicatively performed in three BC patients who were selected based on high CTC counts (identified as epithelial-like CTCs using the RareCyte II platform) and had different hormone receptor status and metastases sites. The 10x Genomics Chromium clustering identified 15 distinct immune cell clusters, which were defined as T cells (five clusters), NK cells (two clusters), B cells (one cluster), macrophages (one cluster), neutrophils (two clusters), and monocytes (four clusters), with platelets being detected within most of these clusters. Importantly, the analysis revealed one cluster of cells with transcriptomic profiles not associated with immune cells, defined as "CTC-candidate" cluster, consisting of 201 cells from both the Lin- and the Lin+ population. This cluster was highly enriched with genes associated with epithelial cells and cell adhesion (e.g., KRT7, 9, 18, and 19, TBX3, TACSTD2, EPCAM, CLDN4, CLDN7, CEACAM6, and MGP), with EPCAM expression being unique to this cluster. It should be noted that many of these genes were expressed in less than $75 \%$ of cells within the cluster, further highlighting the significant degree of heterogeneity of CTCs in BC [18]. Interestingly, several Lin+ cells within the CTC-candidate cluster expressed CD45 either alone or in combination with epithelial markers (EPCAM, TACSTD2, CKs), which is in line with previous evidence that cells co-expressing CD45 and EpCAM or CKs can be identified in the PB of cancer patients [19].

Further analysis of RNA-seq data revealed that the "CTC-candidate" cluster was highly enriched with genes associated with cell adhesion, survival, and cell-to-cell communication. Specifically, genes encoding for claudins and tetraspanins, and other cell adhesion genes, such as MGP, PERP, RAB25, DSP, EMP2, PHLDA2, ERBB2, CEACAM6, and CTNND1, were extensively expressed in this cluster. Moreover, the cluster was enriched with genes coding for signaling proteins (e.g., CRABP2, CAMK2N1, PLK2, RHOV, SPINT1, RALA, and RERG) and proteins implicated in the response to calcium levels (TACSTD2, SRI, S100A16, S100A14, and CRACR2B) and to estrogens (CITED1, CITED4, GATA3, KRT19, CCND1, and IGFBP2). In addition, critical cell proliferation genes (CCND1, ANAPC11, and CDKN1A) and other genes with proliferative roles (TGFB1, ILK, BRK1, CIB1, AREG, and HES1) were also highly expressed. On the other hand, the immune cell 
clusters were highly enriched with genes associated with immune cell activation and inflammatory response, such as CCL5, IL32, and GZMK. Taken together, these findings imply that CTCs show enhanced expression of genes that activate survival signals and, in parallel, modulate their interactions with circulating immune cells. It is critical to note here that the mechanisms promoting the immune evasion capacities of CTCs and the tumor-immune crosstalk in the periphery represent a promising field in cancer research, with potential prognostic and therapeutic implications for BC patients [14,20-23].

The analysis was further focused on genes for transcription factors (TFs), oncogenes, and a series of genes associated with EMT and cancer stem cells (CSCs) within the "CTCcandidate" cluster. Interestingly, Lin- cells demonstrated more heterogeneous and patientspecific patterns, as compared to the Lin+ cells of the cluster. Thus, particular genes for TFs (e.g., LYL1, MAX, and TSC22D), as well as oncogenes (e.g., KIF5BTPM4 and NCOA4) were expressed in Lin-subpopulations, while, in contrast, Lin+ cells were clearly enriched with several TFs and oncogenes. Similarly, the Lin- populations presented a variable expression of epithelial genes (KRT7, 8, 18, and 19, CLDN 4 and 7, EPCAM, TACSTD2, ERBB3, ERBB2, JUP, and CD24) and a remarkably differential expression of several CSC genes, whereas Lin+ fractions were highly homogeneous, according to the expression of genes for mesenchymal-like and CSC phenotypes. These findings strongly imply that distinct cell populations co-exist within the CTC compartment, which could demonstrate different metastatic capacities and resistance or sensitivity to different therapies.

To summarize, Pauken M.C. et al. [17], performed a comprehensive characterization of CTCs in BC patients by employing an unbiased transcriptomic analysis of Lin- and Lin+ cells via scRNA-seq. Their approach allowed the identification of a "CTC-candidate" cluster with a distinct gene expression signature enriched with genes promoting cell adhesion, proliferation, cell-to-cell communication, as well as with genes for TFs, oncogenes, and genes that drive EMT and CSCs, which collectively may foster cell migration and metastatic dissemination. In addition, a significant heterogeneity at the single-cell level was demonstrated for the cells of the cluster. Importantly, the results of the current study question the "classical" CTC definition and point toward the identification of CTCs according to functionalities associated with metastatic potency, rather than according to the expression or absence of specific markers.

The success rate of CTC sequencing is limited by a series of technical issues, such as cell loss, damage of genetic material during isolation, leukocyte contamination, and difficulties in obtaining high quality of sequencing libraries $[9,10]$. Thus, CTC sequencing approaches cannot, as yet, be incorporated into routine CTC assessment, however, they are largely used in translational research for the recognition of biological parameters and pathways specific to metastasis-competent and therapy-resistant CTCs. Although circulating tumor DNA (ctDNA) sequencing is technically less challenging and offers significant information on the genomic and epigenomic landscape of the tumor [24,25], however CTCs can be additionally analyzed at the transcriptome level to uncover DEGs and key regulatory pathways that are active at a given time, thus providing a broad view of the phenotype and dynamics of the tumor [26]. Furthermore, CTC sequencing at the single-cell level can unmask the underlying heterogeneity of the tumor within each individual patient $[27,28]$. Herein, Pauken M.C. et al., in line with a limited number of other studies, underline the importance of performing transcriptomic analyses of CTC s isolated by an epithelial marker independent strategy $[7,11,29,30]$. A further important step should be to delineate the biological function of the CTC subsets identified by Pauken M.C. et al., to explore whether their different transcriptomic patterns are in fact associated with differences in their metastatic potential. In addition, considering that these results were obtained by analyzing a limited number of patients, it is crucial to investigate the distribution of these CTC subpopulations in a larger cohort of BC patients and their associations with patient outcomes.

To conclude, based on this and other reports $[7,31]$ it is likely that the transcriptomic analysis of CTCs will serve in the near future for the discovery of novel prognostic sig- 
natures and of therapeutic strategies that may enhance the implementation of precision medicine and will improve the clinical management of BC.

Conflicts of Interest: The authors declare no conflict of interest.

\section{References}

1. Gupta, G.P.; Massague, J. Cancer metastasis: Building a framework. Cell 2006, 127, 679-695. [CrossRef] [PubMed]

2. Fabisiewicz, A.; Szostakowska-Rodzos, M.; Zaczek, A.J.; Grzybowska, E.A. Circulating Tumor Cells in Early and Advanced Breast Cancer; Biology and Prognostic Value. Int. J. Mol. Sci. 2020, 21, 1671. [CrossRef] [PubMed]

3. Cristofanilli, M.; Pierga, J.Y.; Reuben, J.; Rademaker, A.; Davis, A.A.; Peeters, D.J.; Fehm, T.; Nole, F.; Gisbert-Criado, R.; Mavroudis, D.; et al. The clinical use of circulating tumor cells (CTCs) enumeration for staging of metastatic breast cancer (MBC): International expert consensus paper. Crit. Rev. Oncol. Hematol. 2019, 134, 39-45. [CrossRef] [PubMed]

4. Castro-Giner, F.; Aceto, N. Tracking cancer progression: From circulating tumor cells to metastasis. Genome. Med. 2020, $12,31$. [CrossRef] [PubMed]

5. Lee, J.S.; Magbanua, M.J.M.; Park, J.W. Circulating tumor cells in breast cancer: Applications in personalized medicine. Breast Cancer Res. Treat. 2016, 160, 411-424. [CrossRef] [PubMed]

6. $\quad$ Boral, D.; Vishnoi, M.; Liu, H.N.; Yin, W.; Sprouse, M.L.; Scamardo, A.; Hong, D.S.; Tan, T.Z.; Thiery, J.P.; Chang, J.C.; et al. Molecular characterization of breast cancer CTCs associated with brain metastasis. Nat. Commun. 2017, 8, 196. [CrossRef]

7. Vishnoi, M.; Liu, N.H.; Yin, W.; Boral, D.; Scamardo, A.; Hong, D.; Marchetti, D. The identification of a TNBC liver metastasis gene signature by sequential CTC-xenograft modeling. Mol. Oncol. 2019, 13, 1913-1926. [CrossRef]

8. D' Avola, D.; Villacorta-Martin, C.; Martins-Filho, S.N.; Craig, A.; Labgaa, I.; von Felden, J.; Kimaada, A.; Bonaccorso, A.; Tabrizian, P.; Hartmann, B.M.; et al. High-density single cell mRNA sequencing to characterize circulating tumor cells in hepatocellular carcinoma. Sci. Rep. 2018, 8, 11570. [CrossRef]

9. Miyamoto, D.T.; Zheng, Y.; Wittner, B.S.; Lee, R.J.; Zhu, H.; Broderick, K.T.; Desai, R.; Fox, D.B.; Brannigan, B.W.; Trautwein, J.; et al. RNA-Seq of single prostate CTCs implicates noncanonical Wnt signaling in antiandrogen resistance. Science 2015, 349, 1351-1356. [CrossRef]

10. Ting, D.T.; Wittner, B.S.; Ligorio, M.; Vincent Jordan, N.; Shah, A.M.; Miyamoto, D.T.; Aceto, N.; Bersani, F.; Brannigan, B.W.; Xega, K.; et al. Single-cell RNA sequencing identifies extracellular matrix gene expression by pancreatic circulating tumor cells. Cell Rep. 2014, 8, 1905-1918. [CrossRef]

11. Negishi, R.; Yamakawa, H.; Kobayashi, T.; Horikawa, M.; Shimoyama, T.; Koizumi, F.; Sawada, T.; Oboki, K.; Omuro, Y.; Funasaka, C.; et al. Transcriptomic profiling of single circulating tumor cells provides insight into human metastatic gastric cancer. Commun. Biol. 2022, 5, 20. [CrossRef] [PubMed]

12. Cristofanilli, M.; Budd, G.T.; Ellis, M.J.; Stopeck, A.; Matera, J.; Miller, M.C.; Reuben, J.M.; Doyle, G.V.; Allard, W.J.; Terstappen, L.W.; et al. Circulating tumor cells, disease progression, and survival in metastatic breast cancer. N. Engl. J. Med. 2004, 351, 781-791. [CrossRef] [PubMed]

13. Yu, M.; Bardia, A.; Wittner, B.S.; Stott, S.L.; Smas, M.E.; Ting, D.T.; Isakoff, S.J.; Ciciliano, J.C.; Wells, M.N.; Shah, A.M.; et al. Circulating breast tumor cells exhibit dynamic changes in epithelial and mesenchymal composition. Science 2013, 339, 580-584. [CrossRef]

14. Papadaki, M.A.; Aggouraki, D.; Vetsika, E.K.; Xenidis, N.; Kallergi, G.; Kotsakis, A.; Georgoulias, V. Epithelial-to-mesenchymal Transition Heterogeneity of Circulating Tumor Cells and Their Correlation With MDSCs and Tregs in HER2-negative Metastatic Breast Cancer Patients. Anticancer Res. 2021, 41, 661-670. [CrossRef] [PubMed]

15. Papadaki, M.A.; Stoupis, G.; Theodoropoulos, P.A.; Mavroudis, D.; Georgoulias, V.; Agelaki, S. Circulating Tumor Cells with Stemness and Epithelial-to-Mesenchymal Transition Features Are Chemoresistant and Predictive of Poor Outcome in Metastatic Breast Cancer. Mol. Cancer Ther. 2019, 18, 437-447. [CrossRef] [PubMed]

16. Vishnoi, M.; Peddibhotla, S.; Yin, W.; Scamardo, A.T.; George, G.C.; Hong, D.S.; Marchetti, D. The isolation and characterization of CTC subsets related to breast cancer dormancy. Sci. Rep. 2015, 5, 17533. [CrossRef] [PubMed]

17. Pauken, C.M.; Kenney, S.R.; Brayer, K.J.; Guo, Y.; Brown-Glaberman, U.A.; Marchetti, D. Heterogeneity of Circulating Tumor Cell Neoplastic Subpopulations Outlined by Single-Cell Transcriptomics. Cancers 2021, 13, 4885. [CrossRef] [PubMed]

18. Powell, A.A.; Talasaz, A.H.; Zhang, H.; Coram, M.A.; Reddy, A.; Deng, G.; Telli, M.L.; Advani, R.H.; Carlson, R.W.; Mollick, J.A.; et al. Single cell profiling of circulating tumor cells: Transcriptional heterogeneity and diversity from breast cancer cell lines. PLoS ONE 2012, 7, e33788. [CrossRef]

19. Lustberg, M.B.; Balasubramanian, P.; Miller, B.; Garcia-Villa, A.; Deighan, C.; Wu, Y.; Carothers, S.; Berger, M.; Ramaswamy, B.; Macrae, E.R.; et al. Heterogeneous atypical cell populations are present in blood of metastatic breast cancer patients. Breast Cancer Res. 2014, 16, R23. [CrossRef]

20. Sprouse, M.L.; Welte, T.; Boral, D.; Liu, H.N.; Yin, W.; Vishnoi, M.; Goswami-Sewell, D.; Li, L.; Pei, G.; Jia, P.; et al. PMN-MDSCs Enhance CTC Metastatic Properties through Reciprocal Interactions via ROS/Notch/Nodal Signaling. Int. J. Mol. Sci. 2019, 20, 1916. [CrossRef] 
21. Polioudaki, H.; Mala, A.; Gkimprixi, E.; Papadaki, M.A.; Chantziou, A.; Tzardi, M.; Mavroudis, D.; Agelaki, S.; Theodoropoulos, P.A. Epithelial/Mesenchymal Characteristics and PD-L1 Co-Expression in CTCs of Metastatic Breast Cancer Patients Treated with Eribulin: Correlation with Clinical Outcome. Cancers 2020, 12, 3735. [CrossRef] [PubMed]

22. Papadaki, M.A.; Sotiriou, A.I.; Vasilopoulou, C.; Filika, M.; Aggouraki, D.; Tsoulfas, P.G.; Apostolopoulou, C.A.; Rounis, K.; Mavroudis, D.; Agelaki, S. Optimization of the Enrichment of Circulating Tumor Cells for Downstream Phenotypic Analysis in Patients with Non-Small Cell Lung Cancer Treated with Anti-PD-1 Immunotherapy. Cancers 2020, 12, 1556. [CrossRef] [PubMed]

23. Papadaki, M.A.; Monastirioti, A.; Apostolopoulou, C.A.; Aggouraki, D.; Papadaki, C.; Michaelidou, K.; Vassilakopoulou, M.; Alexakou, K.; Mavroudis, D.; Agelaki, S. TLR4 and pSTAT3 Expression on Circulating Tumor Cells (CTCs) and Immune Cells in the Peripheral Blood of Breast Cancer Patients: Prognostic Implications. Cancers 2022, 14, 1053. [CrossRef]

24. Wan, J.C.M.; Massie, C.; Garcia-Corbacho, J.; Mouliere, F.; Brenton, J.D.; Caldas, C.; Pacey, S.; Baird, R.; Rosenfeld, N. Liquid biopsies come of age: Towards implementation of circulating tumour DNA. Nat. Rev. Cancer 2017, 17, 223-238. [CrossRef] [PubMed]

25. Crowley, E.; Di Nicolantonio, F.; Loupakis, F.; Bardelli, A. Liquid biopsy: Monitoring cancer-genetics in the blood. Nat. Rev. Clin. Oncol. 2013, 10, 472-484. [CrossRef] [PubMed]

26. Rossi, E.; Zamarchi, R. Single-Cell Analysis of Circulating Tumor Cells: How Far Have We Come in the -Omics Era? Front. Genet. 2019, 10, 958. [CrossRef]

27. Miyamoto, D.T.; Ting, D.T.; Toner, M.; Maheswaran, S.; Haber, D.A. Single-Cell Analysis of Circulating Tumor Cells as a Window into Tumor Heterogeneity. Cold Spring Harb. Symp. Quant. Biol. 2016, 81, 269-274. [CrossRef]

28. Keller, L.; Pantel, K. Unravelling tumour heterogeneity by single-cell profiling of circulating tumour cells. Nat. Rev. Cancer 2019, 19, 553-567. [CrossRef]

29. Morrison, G.J.; Cunha, A.T.; Jojo, N.; Xu, Y.; Xu, Y.; Kwok, E.; Robinson, P.; Dorff, T.; Quinn, D.; Carpten, J.; et al. Cancer transcriptomic profiling from rapidly enriched circulating tumor cells. Int. J. Cancer 2020, 146, 2845-2854. [CrossRef]

30. Ring, A.; Campo, D.; Porras, T.B.; Kaur, P.; Forte, V.A.; Tripathy, D.; Lu, J.; Kang, I.; Press, M.F.; Jeong, Y.J.; et al. Circulating Tumor Cell Transcriptomics as Biopsy Surrogates in Metastatic Breast Cancer. Ann. Surg. Oncol. 2022; (ahead of print). [CrossRef]

31. Lang, J.E.; Ring, A.; Porras, T.; Kaur, P.; Forte, V.A.; Mineyev, N.; Tripathy, D.; Press, M.F.; Campo, D. RNA-Seq of Circulating Tumor Cells in Stage II-III Breast Cancer. Ann. Surg. Oncol. 2018, 25, 2261-2270. [CrossRef] 